\title{
Management reorganization of a Radiology ward during the Covid-19 pandemic in a University Hospital structured inpavilions.
}

\section{Guido Marzocchi ( $\sim$ guido.marzocchi@gmail.com )}

Azienda Ospedaliero-Universitaria Policlinico Sant'Orsola Malpighi Bologna https://orcid.org/00000003-2516-2615

\section{Mattia Gentilini}

Azienda Ospedaliero-Universitaria Policlinico Sant'Orsola Malpighi Bologna https://orcid.org/00000003-1288-2381

\section{Alice Gianstefani}

Azienda Ospedaliero-Universitaria Policlinico Sant'Orsola Malpighi Bologna

\section{Fabrizio Giostra}

Azienda Ospedaliero-Universitaria Policlinico Sant'Orsola Malpighi Bologna

\section{Giuliano Orazio Grassi}

Azienda Ospedaliero-Universitaria Policlinico Sant'Orsola Malpighi Bologna

Francesco Monteduro

Azienda Ospedaliero-Universitaria Policlinico Sant'Orsola Malpighi Bologna

\section{Method Article}

Keywords: Management in Radiology, pandemic, COVID-19, Healthcare management

Posted Date: September 22nd, 2020

DOI: https://doi.org/10.21203/rs.3.rs-82055/v1

License: (a) (1) This work is licensed under a Creative Commons Attribution 4.0 International License. Read Full License 


\section{Abstract}

Objective: The aim of the recent management reorganization of the Emergency Room and Radiology ward was to face the recent Coronavirus pandemic in the most rational way possible, to preserve the essential role of these Units in the complex context of a pavillions structured Hospital.

Methods: The most challenging management criticalities posed by the recent Coronavirus pandemic were individually analyzed and dealt with. The pavilions structure of our Hospital, biohazard management, shortage of personnel and shortage or temporary inadequacy of personal protective equipment were addressed as the most urgent and important problems.

Results: A strategic use of the Radiology Information System and Picture Archiving And Communication System (RIS-PACS), dedicated ambulances with specific disinfection procedures, the institution of dedicated pavilions, wards, checkpoints and different paths for COVID-19 (Coronavirs Disease 2019) and non COVID-19 patients, frequent sanification procedures, serological screening for employees and emergency dedicated staff recruitment were put to place as a result of said analysis.

Nevertheless, all of these goals couldn't have been achieved without a constant sense of responsibility of the employees involved.

Conclusions: A rational analysis of the most critical problems associated with a pandemic is vital to provide a fast and effective response, and represents a valuable wealth of experience in case of future pandemics.

\section{Background}

In December 2019 in Wuhan Urban Area, China, a new coronavirus (SARS-CoV-2 or Severe Acute Respiratory Syndrome Coronavirus 2) was identified as the responsible for the pulmonary infection today known as COVID-19.

SARS-CoV-2 is characterized by high contagiousness and the ability to spread by air through droplets $>5$ $\mu \mathrm{m}$, through direct contact or with contaminated material [1-3]; it has a mean incubation time of 3 days (range 0-24) with potential transmission in the asymptomatic period of the disease [2,4].

This illness presents with symptoms such as fever, malaise, dry cough, dyspnoea, diarrhea, skin rashes, conjunctivitis and respiratory distress, with a more severe clinical course in elderly patients with comorbidities, causing respiratory failure due to diffuse alveolar damage, multi organ failure, shock and possibly death [5-7]. Patients who suffer from COVID-19-associated ARDS who have both a reduction in 
respiratory system compliance and an increased D-dimer concentrations are subject to higher mortality rates [8].

On January 30th, 2020 WHO declared the pandemic as a global emergency. On 21st of February 2020 the pandemic reached Italy, which until that moment counted only 3 cases.

Thus, it became necessary for radiological wards to put in place all of the possible prevention measures to prevent the spread of the infection between hospital personnel and patients (transiting or hospitalized).

To date (June 25th) the pandemic struck with more than 13 million cases worldwide. Italy, with 239.000 cases, is the second most affected country in Europe and the ninth in the world. Emilia Romagna is the third most affected region with 28.304 cases and 4245 victims. In Bologna province until the 25th of June 5129 cases were recorded.

To date the transmissibility ratio " $\mathrm{R} 0$ " in our region is 0,49 .

During the period between $1 / 3 / 2020$ and $31 / 5 / 2020$ the number of patients who were evaluated in the Emergency Room of the University Hospital Policlinico Sant'Orsola-Malpighi and had the word "COV" included in the discharge diagnosis was 2.784. The Emergency Room had a mean of 50 extra patients every day, with peaks reaching 100 extra patients a day; around 4500 radiological exams were performed during the pandemic. Patients that had to perform nasopharyngeal swabs have undergone -depending on the severity of the symptoms- chest X-ray, Lung ultrasound and around 900 Chest CT, according to the Italian Society of Medical Radiology guidelines (Utilizzo della diagnostica per immagini nei pazienti COVID-19 intersocietal document SIRM, SIUMB, FISM). The sharp increase of the requests of radiological examinations during the pandemic is easily understandable if one consider that the RT-PCR genomic test (real-time reverse transcription polymerase chain reaction), to date the gold standard for COVID-19 diagnosis, poses great management issues because of a sensibility around $60 \%[2,3]$ with a significant risk of false negative patients that could be hospitalized in non-dedicated wards, representing a source of infection.

Possible causes of this low sensibility could be a low viral load, inappropriate execution of the swab, the timing of the execution within the course of the disease or laboratory issues [9].

Furthermore, the test procedure requires time, not always easily available during the first moments of a serious pandemic.

Instead, it has been proven that the sensibility of High Resolution Computed Tomography (HRCT) scan after at least two days from the onset of symptoms can be even higher than that of the genomic test [10], and because of this, reliability imaging has won a pivotal role in both diagnosis and follow up of the disease through several modalities:

CHEST X-RAY 
Cozzi et al. recently showed [11] that although $5,6 \%$ of patients positive to PCR have a negative chest Xray (CXR) (probably patients in the earlier stages of the disease or asymptomatic cases) the majority of CXR shows findings that help determine the severity of the disease and address the subsequent management of the patient. Most frequent findings are consolidation ( $57,7 \%$ of cases), ground glass opacities $(62,8 \%)$, reticulonodular opacities $(66,6 \%)$ and nodules $(23,5 \%)$.

The distribution of these findings is greater peripherally $(57,7 \%)$ and in the lower lobes $(58,5 \%)$, and a bilateral involvement $(69,2)$ is more frequent than the unilateral (Figure 1).

This kind of radiological study has a sensibility of $67 \%$ and the intra observer reproducibility is excellent.

Several scores can be used in the evaluation of the CXR performed in the Emergency Room, moreover with good correlation with the subsequent course of the disease and the probability of need of Intensive Care support.

HRCT

Chest CT plays a key role not only in the diagnosis of the disease (especially when dealing with RT-PCR false negatives), but also in evaluating the course of the disease and in assessing its severity.

Typical findings of COVID-19 [12] are bilateral, peripheral ground glass opacities with or without consolidations, especially with posterior distribution. Consolidations, linear opacities, crazy paving pattern, reversed halo and ectatic vessels within the consolidations are found in more advanced stages of the disease (Figure 2).

The Radiologist should anyway be very careful and always check clinical and laboratory findings, since the features described can have several aspects in common with influenza and parainfluenza viruses, adenovirus, respiratory syncytial virus, rhinovirus, metapneumovirus, other less common agents and non infectious diseases (cryptogenic organizing pneumonia, drugs reaction...).

Using RT-PCR as a reference Ai et al [10] showed that computed tomography has a high sensibility (97\%), albeit with a low specificity (25\%), and with an accuracy of $68 \%$ for COVID-19 diagnosis; therefore typical findings at chest CT can help in pointing out false negatives although as said with constant attention to possible differential diagnoses.

What's more, deep learning models applied to CT imaging could be useful to increase specificity and sensitivity to help the Radiologist with the differential diagnosis with community aquired pneumonia and other lung diseases [13].

\section{LUNG ULTRASOUND}

Lung ultrasound (LUS) is a valuable tool for Emergency Room physicians on the first line, especially when the transfer of an infected patient is particularly difficult, or when it is advisable to avoid ionizing radiations, and also in certain second and third world settings, where it has a much easier availability if 
compared to computed tomography. Furthermore, the time needed to sanitize the equipment is less if compared to computed tomography.

A recent meta analysis [14] showed that the majority of COVID-19 patients have pathological findings at LUS. The most frequent is interstitial involvement as demonstrated by a B-lines pattern (figure 3 ), followed by pleural thickening and irregularities, consolidations and seldomly pleural effusions.

Although LUS is significantly operator dependent and reserved for specialized and trained personnel, it can be useful when performing triage, diagnosis and follow up [15].

\section{Methods}

A few days after the arrival of the pandemic in Italy it was decided to analyze and assess the main issues posed by a large hospital structured in pavilions, in order to respond to these challenges in the most efficient and fast way possible.

Our findings consisted of the pavillions structure of the hospital, management of the biological hazard in regards to both personnel and patients, adjustments in staff numbers to face the pandemic in a context of incomplete turn over and the shortage or temporary inadequacy of personal protective equipment (Table 1).

Table 1: The analyzed criticalities and their applied solutions.

\begin{tabular}{|l|l|}
\hline \multicolumn{1}{|c|}{ Criticalities } & \multicolumn{1}{c|}{ Solution } \\
\hline Pavilions structured Hospital & Strategic use of the RIS-PACS system \\
\hline & $\begin{array}{l}\text { Dedicated ambulances with specific disinfection } \\
\text { procedures }\end{array}$ \\
\hline & Dedicated pavilions \\
\hline Biohazard management & Creation of dedicated pavilions and wards \\
\hline & $\begin{array}{l}\text { Creation of different paths for COVID-19 and non } \\
\text { COVID-19 patients }\end{array}$ \\
\hline & $\begin{array}{l}\text { Frequent and specified sanification procedures of } \\
\text { radiological equipment and relative rooms }\end{array}$ \\
\hline & Institution of checkpoints \\
\hline Shortage of personnel & Serological screening for employees \\
\hline $\begin{array}{l}\text { Shortage or temporary inadequacy } \\
\text { of personal protective equipment }\end{array}$ & Emergency dedicated staff recruitment \\
\hline
\end{tabular}

\section{Results}

All the criticalities analized were faced as follows. 


\section{Pavilions structured Hospital}

The complex reality of our University Hospital is structured in 30 pavilions (1680 beds with 857 physicians, of which 222 with University contracts, and 58 Radiologists) and routinely needs regular assessments of workflows which result from increases or decreases of the number and type of required radiological examination (about 335.000 radiological studies per year).

Demand-side responses are often required, with rearrangements of beds and wards with their relocation.

Radiology department itself is also composed of several sections (Cardio-thoracic, Specialized, Emergency, Pediatric, Senology, Interventional), which merged in a single operative unit to optimize and rationalize available human resources.

The complexity of a pavilion structure brings logistical and organizing issues, first of all patients transport and personnel movement.

\subsection{Personnel movement}

In a context of increased risks in transporting patients and increased biohazard the RIS-PACS system played a fundamental role enabling Radiologists, always respecting ISTISAN (Istituto Superiore di Sanità) $10 / 44$ ministerial guidelines on teleradiology, to work simultaneously on exams from patients in different pavilions.

In this way conventional radiographs and non-enhanced CT scan could be dealt with in less time without physically moving to other buildings and with less stress for the medical personnel, often enduring long work shifts.

\subsection{Patient transport}

In the first stages of the pandemic patients were transported between buildings with ambulances, sanitized after each transfer.

With the exponential growth of cases however, it became evident that, to avoid delays in the management of the patients flow due to the inevitable time of disinfection of the vehicles, and to limit the number of transports -source of potential risk for patients and exposure for health workers- it would have been necessary to set up at least two pavilions dedicated to the emergency: the first already equipped with a computed tomography and X-ray machine and a second one, normally used as Emergency Room, Emergency Medicine and Surgery and Internal Medicine. In these buildings it would have been possible and convenient to perform X-ray, computed tomography scans and ultrasound on known or suspect COVID-19 patients, coming from the Emergency Room, from COVID-19 wards or from the intensive care unit.

\section{Biohazard management}


The diagnostic and clinical activities which take place in hospitals and health facilities pose a very high risk of infection to the health workers.

In our radiological department we promptly developed the following measures which aimed to minimize the biological hazard. Our main objective has been the confinement of the infected patients in order to protect both the patients accessing the ER, and the health care operators, which represent the most precious asset in facing a pandemic.

2.1: The first step in containing the biological hazard has been the establishment of dedicated departments and pavilions. As recently stated by the literature $[16,17,18]$ this precaution, together with the creation of different paths for infected or suspected positive patients, is essential for the decrease of the potential biological hazard.

Our hospital, following the evolution of the epidemic and according to the Emilia-Romagna regional directives, with an extraordinary plan, and in close coordination with the Bologna USL Agency, has implemented the Intensive Care Units services and converted the regular hospital departments into dedicated COVID-19 departments.

As aforementioned, we provided to identify and set up a small radiological department (equipped with an X-ray machine and a Computed Tomography) into the pavilion dedicated to the confirmed or suspected COVID-19 patients incoming from the ER.

With the progression of the epidemic and the resulting increase of the cases, it became necessary to identify into the ER pavilion a further structure dedicated to host confirmed COVID-19 and suspected COVID-19 departments.

2.2: The institution of different accessing paths to the ER: In the attempt of reducing as much as possible the contact between suspected COVID-19 patients and regular ER patients, since march 18th 2020, the ER has also been divided into two different areas, each one dedicated to a first evaluation of the patient and its proper diagnostic-therapeutic path. During the first part of the pandemic, where the majority of patients that were accessing the ER were suspected COVID-19 patients, they were handled into the main area of the ER which is the High-Intensity Area and the Temporary Observation Area (Purple Track). Meanwhile, the non-COVID-19 patients were handled into a smaller area of the ER, which is the LowIntensity Area and the Orthopedic Area which has been reconverted to handle the red/yellow codes (Orange Track) (Figure 4).

During the second part of the pandemic instead, when the number of regular ER (non-COVID-19) patients was much higher compared to the number of suspected COVID-19 patients, the two areas were inverted.

The division of the patients as has been described and the decrease of the contacts between the two categories of patients has been possible also thanks to a PRE-TRIAGE tent placed in front of the main access to the ER pavilion, in which the patient was instructed to sanitize his/her hands with hydroalcoholic gel, to wear a surgical mask, to undergo body temperature detection and had to answer a 
questionnaire that would assess his/her risk of being positive to COVID-19. After this first "pre-triage" the patient was then designated to the Purple or Orange Track. This very same division between the two kinds of patients has also been carried out to all those ones who were taken to the ER by ambulance, which underwent "PRE-TRIAGE" already on board of the vehicle, and subsequently were designated to one of the two paths. The ER health care personnel has also been reorganized and implemented so that the two different teams of the two tracks were separated as well. Furthermore, during a two-months period (April the 2nd until June the 5th) was also working the so called "infectious disease fast-track". This was a dedicated path for patients with suspected COVID-19 disease, a breath frequency $<18 / \mathrm{min}$ and $\mathrm{SpO} 2>95 \%$. The patients could undergo a triage through either direct access or after their family doctor had reserved them a visit in the ER. This fast-track included an electrocardiogram, a blood gas analysis and a "sit to stand" test, followed by a visit from an ER doctor which would also perform a lung ultrasound. After this whole set of exams, the infectious disease specialist would decide whether to dismiss or to admit the patient.

2.3 All the CT machines of the hospital have been divided into "dirty" and "clean", in order to minimize the contact between infected and non-infected patients and to reduce the number of required disinfections. At first, with the need of 3 to 5 chest-CT exams per day, we established a dedicated CT machine situated in the dedicated COVID-19 pavilion. As the epidemic progressed though, and the number of positive or suspected positive patients coming from the ER pavilion to the COVID-19 pavilion to undergo a CT scan kept growing, the time required to sanitize the ambulances became impossible to sustain. It must also be taken into account that the technical time needed to perform a scan was increasing due to the distancing between patients and the disinfection and ventilation of the CT-machine room. The importance to ventilate a confined environment such as the CT room has been shown in the literature [19], since it has been proven that the virus expired by infected individuals can be dispersed through the airflows that happen into a small and closed environment even without procedures that generate aerosol particles. With a further progression of the epidemic has then been established a second CT-machine dedicated to the COVID-19 patients at the first floor of the ER pavilion (reducing the need of transportation for the suspect patients), while the dedicated ER-CT machine has been kept for the regular ER activities for other emergencies. It has also been assigned a dedicated Radiologist who had to report exclusively the exams of COVID-19 patients that were executed during the night, while another Radiologist was instead working in the regular night shift in the ER. We also took into account some special occurrences, such as:

- for those patients suspected of having COVID-19 who were in a life-threatening condition, the regular ER CT machine has been used, since it was the closest and fastest available machine, and was later sanitized.

- in the rare necessity of urgent need for a CT scan for both infected and non-infected patients, the first ones were scanned in a batch, and accurate disinfection of the equipment and room followed.

- whenever a regular ER patient would have a CT scan suspect or positive for COVID-19 disease, the CT machine and its room were sanitized, even before the results of the RT-PCR.

\subsection{Frequent and specific sanitation procedures}


Sanitization consists of all the procedures intended to sanitize certain environments and equipment through cleaning and disinfection. The cleaning's aim is to remove all the dust, dirty material or grime from all the surfaces, objects and different environments. Disinfection instead includes all the procedures aiming to kill or inactivate pathogenic microorganisms.

It has been shown by a number of studies about other coronaviruses that this family of viruses is more stable in confined environments compared to other viruses with envelope [20]. It is then fundamental to take all the necessary precautions to prevent a further spread of the virus with prompt and specific disinfection procedures of the surfaces and environments.

The SARS-COV-2 virus is susceptible to the most commonly used disinfectant substances, which inactivate it with rapid effect. Ethyl alcohol-based disinfectant with a dilution of at least $70 \%$, or hydroxide peroxide disinfectant with a dilution of at least $0,5 \%$ and sodium hypochlorite disinfectant with a dilution of at least $0,1 \%$ have been found effective [21]. Overall, SARS-COV-2 is stable in a stable environment, but is also highly susceptible to the standard disinfecting procedures [22], therefore it is possible to utilize all the products indicated by the standard ISO EN 14476. On the other hand, it hasn't been shown to date any effectiveness in the disinfecting power of ozone on surfaces contaminated by SARS-CoV-2. In the health sector, the disinfection and decontamination procedures and a proper hand washing are key elements to reduce the risk of indirect contamination. At first, the cleaning process of all the radiological equipment was assigned to the usual cleaning team. To avoid excessive delays though, during the peak of the pandemic, this task has been assigned to the radiology personnel after every single exam on a suspected or positive COVID-19 patient. As disinfectants, we tried to use mostly non-corrosive substances to avoid any damage to the equipment, being especially aware of possible corrosion of the machines or electrical damages if any substance would have leaked inside the machines. For this reason, when possible, the radiological equipment was shut down before the disinfection procedures. Cleaning of the environments (floors, windows, chairs and so on) has been taking place at intervals of 5 to 6 hours as suggested by the WHO [21], or more often if specifically asked by the medical personnel. The single-use devices utilized in disinfection procedures have always been disposed of into proper "high biological risk trash cans" to be then stored as highly infective and dangerous material. Furthermore, we also proceeded to ventilate all the environments before and after the cleaning procedures. In our experience, we did not utilize any UV or ozone devices, because of not proven effectiveness.

2.5 The establishment of checkpoints at the main entrance of the pavilions with the highest number of accesses, where people were briefly interviewed about any possible COVID-19 symptom. At the entrance of all the pavilions was imposed to wear a face mask and to sanitize the visitor's hands with hydroalcoholic gel. The surgical mask worn by COVID-19 infected patients prevents the emission of droplets reducing in a substantial way the risk of infection during interpersonal contact [23].

2.6 Serological tests for all the hospital employees All the hospital employees underwent at least two serological tests 6 weeks apart in search for IgG and IgM. This occurred with two aims: firstly for an epidemiological screening, secondarily to diagnose possible asymptomatic or mild infections, so that we 
could prevent a further spread of the disease. In case of a IgM positivity the employee quickly underwent a PCR test for confirmation. In case of suspected symptoms during the pandemic, the employees were quickly submitted to a PCR test and, if needed, a chest X-ray or a chest-CT scan.

\section{Shortage of personnel}

In order to face such a massive and unpredictable amount of patients who required imaging exams new Medical Doctors and Radiology Technicians were recruited for a 6 months term, an opportunity which regarded both Radiologists and residents in Radiology from the fourth and third (last and penultimate) year of residency.

\section{Shortage or temporary inadequacy of personal protective equipment}

At the beginning of the epidemic we also had to face a severe shortage of Personal Protective Equipment (PPE), especially FFP2 and FFP3 masks, protective glasses and goggles, coats, shirts, caps and overshoes. As it has emerged by a recent investigation, we shared this experience with the majority of the other radiologists of the country, especially with those in the southern part [24]. Also the task of surveillance for the safety of the hospital personnel has been very challenging, especially due to the high variety of symptoms of the COVID-19 infection and a large amount of asymptomatic and mildly symptomatic patients. Nevertheless, the personnel has faced the pandemic with the awareness of a high personal-risk, but they did so for the safety of their own community, and with a great sense of responsibility.

\section{Conclusion}

The challenge posed by the recent (and far from being over) pandemic has shown, now more than ever, how a close interaction between the personnel of the ER, the radiology department, the internal medicine department and the Intensive Care Units departments is crucial to promptly recognize, treat and follow such a complex and dangerous disease.

The strategic use of a modern RIS - PACS system, institution of different accessing paths, frequent and specific sanitation procedures, the establishment of checkpoints at the main entrance of pavilions, serological tests for all the hospital employees and hiring of new personnel have all been key strategies to keep working the complex and potentially fragile system which is a large hospital.

To all these measures we must add the undeniable sense of responsibility and sacrifice of our personnel, which, despite the dangers it had to face, has always kept doing its work despite the temporary lack of PPE.

The radiology, in all of its declinations, has always been a fundamental tool for diagnosis and follow-up of the majority of the diseases, both in emergency-urgency medicine and in elective medicine, and it is essential that it keeps a proper and rational operativeness, even and especially during a crisis. 
The measures that we applied to face the COVID-19 pandemic require a strict application of the aforementioned protocols and close cooperation between radiologists, nurses, radiologist technicians and all the remaining personnel involved. These solutions posed to such a challenge as this pandemic has been, allowed us to handle at our best the emergency, even if sometimes it took our hospital to its limit. And yet these managing efforts must not be considered as a single event, but rather as an important wealth of experience in the very possible eventuality of a second wave, even if it shows in isolated focuses, or also in case of a different pandemic.

\section{Abbreviations}

COVID-19: Corona Virus Disease 2019

CT: Computed Tomography

CXR: Chest X-ray

ER: Emergency Room

HRCT: High Resolution Computed Tomography

LUS: Lung Ultrasound

ISTISAN: Istituto Superiore di Sanità

PPE: Personal Protective Equipment

RIS-PACS: Radiology Information System and Picture Archiving And Communication System

RT-PCR: Real-Time reverse transcription Polymerase Chain Reaction

SARS-COV-2: Severe Acute Respiratory Syndrome Coronavirus-2

WHO: World Health Organization

\section{Declarations}

Funding This study was not supported by any funding.

Conflicts of interest/Competing interests The authors declare that they have no conflict of interest.

Ethics approval Not applicable

Consent to participate Not applicable

Consent for publication All Authors give their consent for publication of this paper. 
Availability of data and material Not applicable

Code availability Not applicable

\section{References}

1. Ng MY, Lee EYP, Yang J, Yang F, Li X, Wang H, Lui MMS, Lo CSY, Leung B, Khong PL, Hui CKM, Yuen KY, Kuo MD (2020) Imaging Profile of the COVID-19 Infection: Radiologic Findings and Literature Review. Radiology: Cardiothoracic Imaging, 2(1), p.e200034. Doi: 10.1148/ryct.2020200034

2. Yang Y, Lu Q, Liu M, Wang Y, Zhang A, Jalali N, Dean N, Longini I, Halloran M, Xu B, Zhang X, Wang L, Liu W, Fang L (2020) Epidemiological and clinical features of the 2019 novel coronavirus outbreak in China. medRxiv 2020.02.10.20021675; doi: https://doi.org/10.1101/2020.02.10.20021675

3. Pan F, Ye T, Sun P, Gui S, Liang B, Li L, Zheng D, Wang J, Hesketh RL, Yang L, Zheng C (2020) Time Course of Lung Changes at Chest CT during Recovery from Coronavirus Disease 2019 (COVID-19). Radiology 295(3):715-721. https://doi.org/10.1148/radiol.2020200370

4. Chung M, Bernheim A, Mei X, Zhang N, Huang M, Zeng X, Cui J, Xu W, Yang Y, Fayad ZA, Jacobi A, Li K, Li S, Shan H (2020) CT Imaging Features of 2019 Novel Coronavirus (2019-nCoV). Radiology 295(1):202-207. https://doi.org/10.1148/radiol.2020200230

5. Chen N, Zhou M, Dong X, Qu J, Gong F, Han Y, Qiu Y, Wang J, Liu Y, Wei Y, Xia J, Yu T, Zhang X, Zhang L (2020) Epidemiological and clinical characteristics of 99 cases of 2019 novel coronavirus pneumonia in Wuhan, China: a descriptive study. Lancet (London, England) 395(10223):507-513. https://doi.org/10.1016/S0140-6736(20)30211-7

6. Wang D, Hu B, Hu C, Zhu F, Liu X, Zhang J, Wang B, Xiang H, Cheng Z, Xiong Y, Zhao Y, Li Y, Wang X, Peng Z [published online: February 07, 2020] Clinical Characteristics of 138 Hospitalized Patients With 2019 Novel Coronavirus-Infected Pneumonia in Wuhan, China. JAMA https://doi.org/10.1001/jama.2020.1585

7. Huang C, Wang Y, Li X, Ren L, Zhao J, Hu Y, Zhang L, Fan G, Xu J, Gu X, Cheng Z, Yu T, Xia J, Wei Y, Wu W, Xie X, Yin W, Li H, Liu M, Xiao Y, Gao H, Guo L, Xie J, Wang G, Jiang R, Gao Z, Jin Q, Wang J, Cao B (2020) Clinical features of patients infected with 2019 novel coronavirus in Wuhan, China. Lancet (London, England) 395(10223):497-506. https://doi.org/10.1016/S0140-6736(20)30183-5

8. Grasselli G, Tonetti T, Protti A, Langer T, Girardis M, Bellani G, Laffey J, Carrafiello G, Carsana L, Rizzuto C, Zanella A, Scaravilli V, Pizzilli G, Grieco DL, Meglio L, De Pascale G, Lanza E, Monteduro F, Zompatori M, Seccafico C (Published Online 2020/08/01) Pathophysiology of COVID-19-associated acute respiratory distress syndrome: a multicentre prospective observational study. The Lancet Respiratory Medicine. https://doi.org/10.1016/S2213-2600(20)30370-2 
9. Fang Y, Zhang H, Xie J, Lin M, Ying L, Pang P, Ji W (2020) Sensitivity of Chest CT for COVID-19: Comparison to RT-PCR. Radiology 296(2):E115-E117. https://doi.org/10.1148/radiol.2020200432

10. Ai T, Yang Z, Hou H, Zhan C, Chen C, Lv W, Tao Q, Sun Z, Xia L (2020) Correlation of Chest CT and RTPCR Testing for Coronavirus Disease 2019 (COVID-19) in China: A Report of 1014 Cases. Radiology 296(2):E32-E40. https://doi.org/10.1148/radiol.2020200642

11. Cozzi D, Albanesi M, Cavigli E, Moroni C, Bindi A, Luvarà S, Lucarini S, Busoni S, Mazzoni LN, Miele V. (2020) Chest X-ray in new Coronavirus Disease 2019 (COVID-19) infection: findings and correlation with clinical outcome. La Radiologia medica 125(8):730-737. https://doi.org/10.1007/s11547-02001232-9

12. Carotti M, Salaffi F, Sarzi-Puttini P, Agostini A, Borgheresi A, Minorati D, Galli M, Marotto D, Giovagnoni A (2020) Chest CT features of coronavirus disease 2019 (COVID-19) pneumonia: key points for radiologists. La Radiologia medica 125(7):636-646. https://doi.org/10.1007/s11547-020-01237-4

13. Neri E, Miele V, Coppola F, Grassi R. (2020) Use of CT and artificial intelligence in suspected or COVID19 positive patients: statement of the Italian Society of Medical and Interventional Radiology. La Radiologia medica 125(5):505-508. https://doi.org/10.1007/s11547-020-01197-9

14. Mohamed MFH, Al-Shokri S, Yousaf Z, Danjuma M, Parambil J, Mohamed S, Mubasher M, Dauleh MM, Hasanain B, AlKahlout MA, Abubeker IY. (2020) Frequency of Abnormalities Detected by Pointof-Care Lung Ultrasound in Symptomatic COVID-19 Patients: Systematic Review and Meta-Analysis. The American journal of tropical medicine and hygiene 103(2):815-821. https://doi.org/10.4269/ajtmh.20-0371

15. Di Serafino M, Notaro M, Rea G, lacobellis F, Delli Paoli V, Acampora C, lanniello S, Brunese L, Romano L, Vallone $\mathrm{G}$ (2020) The lung ultrasound: facts or artifacts? In the era of COVID-19 outbreak. La Radiologia medica 125(8):738-753. https://doi.org/10.1007/s11547-020-01236-5

16. Gutzeit A, Li Q, Matoori S, Li B, Wang L (2020) What can European radiologists learn from the outbreak of COVID-19 in China? A discussion with a radiologist from Wuhan. European radiology 30(7):3609-3611. https://doi.org/10.1007/s00330-020-06841-6.

17. Ding J, Fu H, Liu Y, Gao J, Li Z, Zhao X, Zheng J, Sun W, Ni H, Ma X, Feng J, Wu A, Liu J, Wang Y, Geng P, Chen $Y$ (2020) Prevention and control measures in radiology department for COVID-19. European radiology 30(7):3603-3608. https://doi.org/10.1007/s00330-020-06850-5

18. Politi LS, Balzarini L (2020) The Radiology Department during the COVID-19 pandemic: a challenging, radical change. European radiology 30(7):3600-3602. https://doi.org/10.1007/s00330020-06871-0 
19. Chia PY, Coleman KK, Tan YK, Ong SWX, Gum M, Lau SK, Lim XF, Lim AS, Sutjipto S, Lee PH, Son TT, Young BE, Milton DK, Gray GC, Schuster S, Barkham T, De PP, Vasoo S, Chan M, Ang BSP, Tan BH, Leo YS, Ng OT, Wong MSY, Marimuthu K, Lye DC, Lim PL, Lee CC, Ling LM, Lee L, Lee TH, Wong CS, Sadarangani S, Lin RJ, Ng DHL, Sadasiv M, Yeo TW, Choy CY, Tan GSE, Dimatatac F, Santos IF, Go CJ, Chan YK, Tay JY, Tan JY, Pandit N, Ho BCH, Mendis S, Chen YYC, Abdad MY, Moses D (2020) Detection of air and surface contamination by SARS-CoV-2 in hospital rooms of infected patients. Nature communications 11(1):2800. https://doi.org/10.1038/s41467-020-16670-2

20. Wolff MH, Sattar SA, Adegbunrin O, Tetro J (2005) Environmental survival and microbicide inactivation of coronaviruses. In: Schmidt A, Weber O, Wolff MH (eds) Coronaviruses with Special Emphasis on First Insights Concerning SARS. Birkhäuser Advances in Infectious Diseases BAID. Birkhäuser Basel. https://doi.org/10.1007/3-7643-7339-3_10

21. Cleaning and disinfection of environmental surfaces in the context of COVID-19, WHO Interim guidance, WHO reference number: WHO/2019-nCoV/Disinfection/2020.1

22. Chin AWH, Chu JTS, Perera MRA, Hui KPY, Yen HL, Chan MCW, Peiris M, Poon LLM. (2020) Stability of SARS-CoV-2 in different environmental conditions. The Lancet. Microbe 1(1):e10. https://doi.org/10.1016/S2666-5247(20)30003-3

23. Advice on the use of masks in the context of COVID-19, WHO interim guidance, WHO reference number: WHO/2019-nCov/IPC_Masks/2020.4

24. Albano D, Bruno A, Bruno F, Calandri M, Caruso D, Clemente A, Coppolino P, Cozzi D, De Robertis R, Gentili F, Grazzini I, Jannone ML, Liguori C, Natella R, Pace G, Posa A, Scalise P, Accarino B, Bibbolino C, Barile A, Grassi R, Messina C [published online: July 14, 2020] Impact of coronavirus disease 2019 (COVID-19) emergency on Italian radiologists: a national survey. European radiology https://doi.org/10.1007/s00330-020-07046-7

\section{Figures}




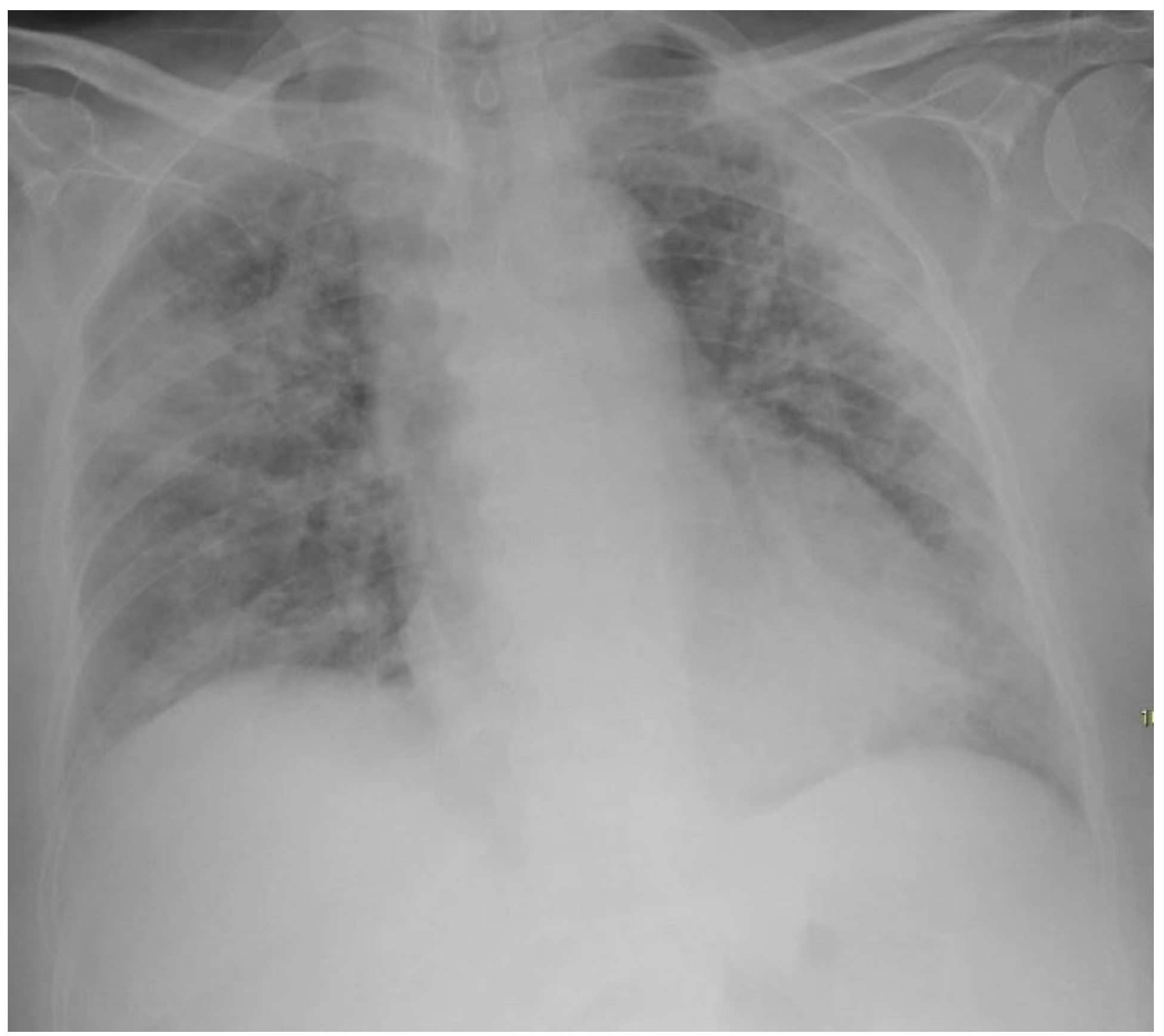

\section{Figure 1}

bilateral, mostly peripheral consolidations and ground glass opacities in a COVID-19 patient 

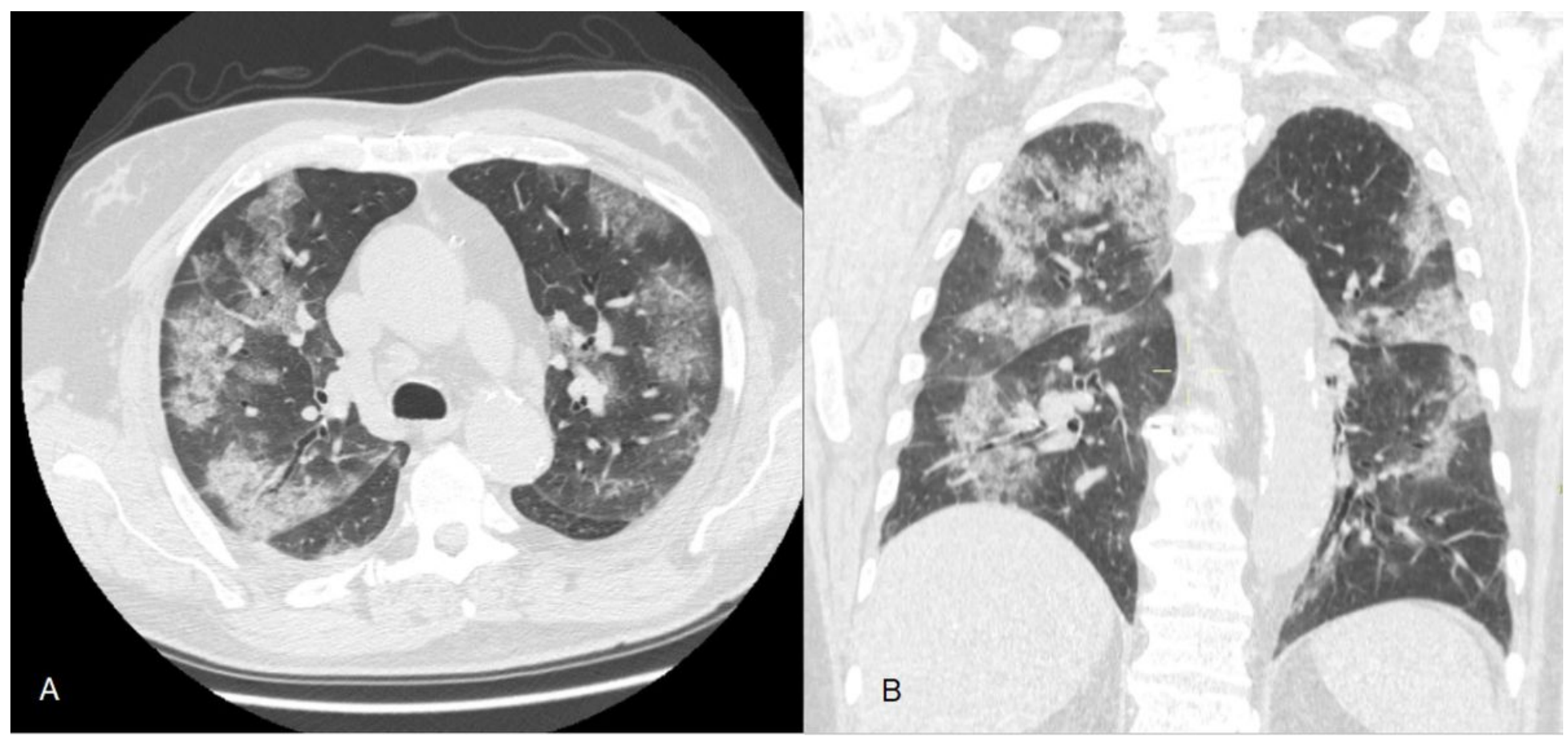

Figure 2

bilateral, peripheral diffuse ground glass opacities and crazy paving pattern without significant pleural effusion (A: axial, B: coronal planes). 


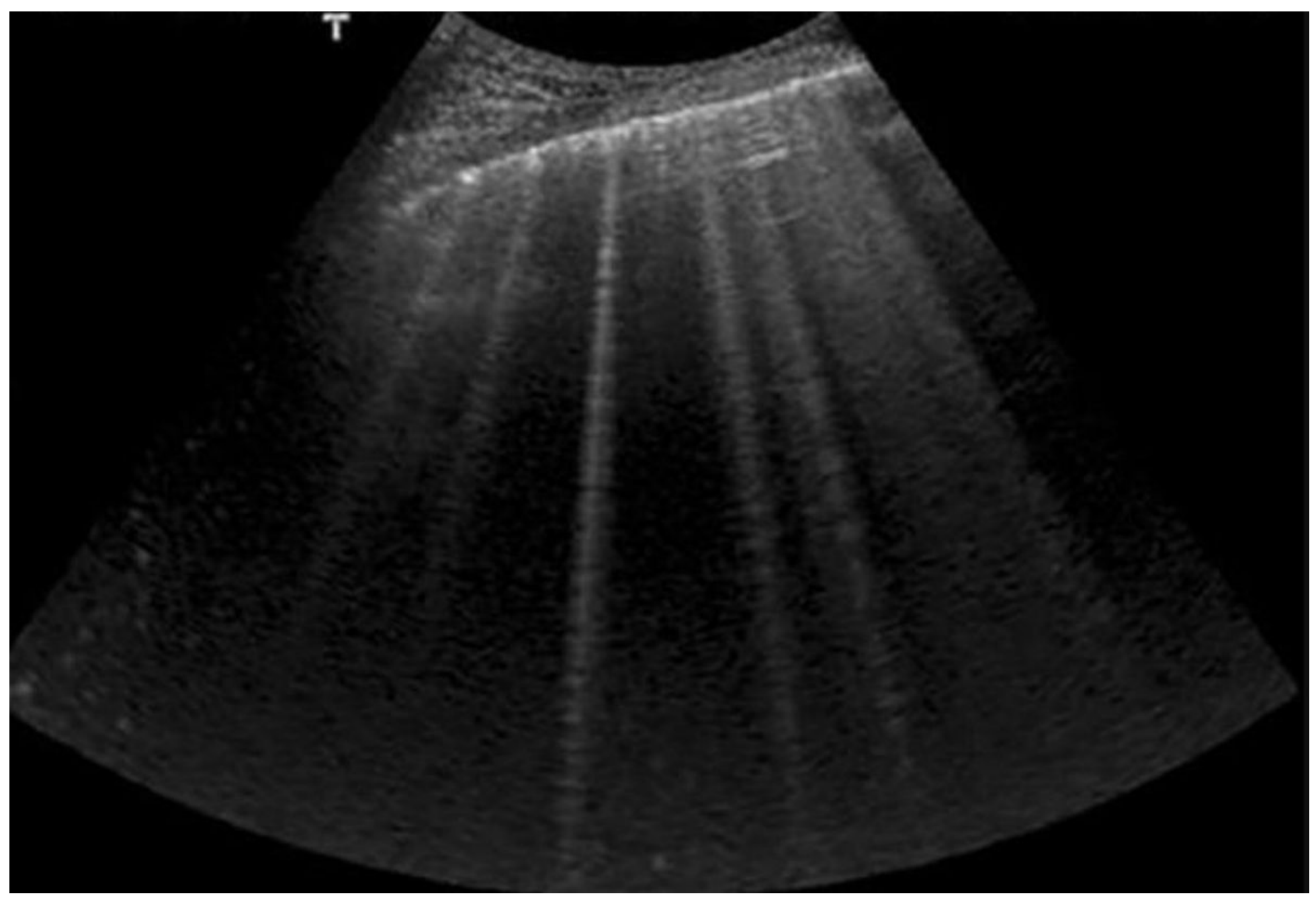

Figure 3

coalescent vertical artifacts (B-lines) erasing normal A-lines. 


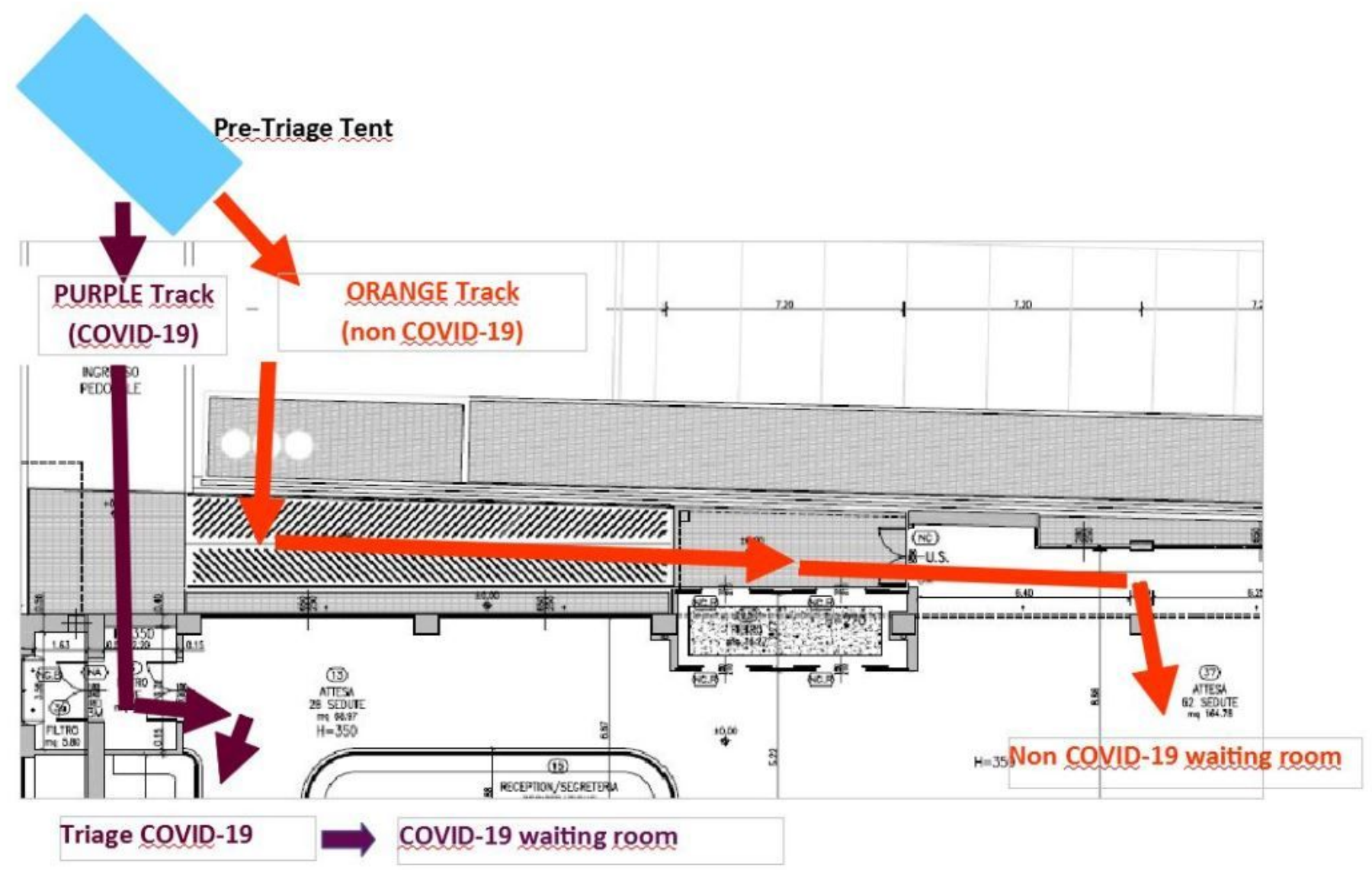

Figure 4

the different tracks to reduce possible contact between suspected COVID-19 patients and other ER patients. 